\title{
Analysis of conjugate heat transfer of a thermally developing flow in a microtube
}

\author{
Khalid M. RAMADAN* \\ *University of Sharjah, Department of Mechanical and Nuclear Engineering, Sharjah, 27272, UAE \\ E-mail: kramadan@sharjah.ac.ae
}

Received: 1 June 2019; Revised: 10 September 2019; Accepted: 8 October 2019

\begin{abstract}
Conjugate heat transfer in a microtube gas flow with constant wall temperature boundary condition is studied numerically, taking into consideration the effects of the shear work, axial conduction rarefaction, pressure work and viscous dissipation. Analytical solutions for the case with non-zero Brinkman number are also obtained. The effect of the tube wall thickness and the wall thermal conductivity on Nusselt number, inner wall heat flux, bulk gas temperature, inner wall temperature and other heat transfer characteristics are analyzed. Comparisons with the standard zero wall thickness case are also presented. The results illustrate the significance of the wall heat conduction on the thermal entrance length and the heat transfer characteristics in the microtube in the thermally developing and the fully developed flow regions, and show the deviation from those with zero wall thickness. The analysis shows that in the thermally fully developed region, the Nusselt number and the heat flux at the inner wall are however, not influenced by the tube wall heat conduction. Heat exchange between the gas and the wall is shown to take place only in the thermal entrance region.
\end{abstract}

Keywords : Conjugate, Thermal entrance length, Shear work, Flow work, Viscous dissipation

\section{Introduction}

In engineering applications involving convective heat transfer in channels, where the channel wall thickness is much smaller than the channel hydraulic diameter, the conduction heat transfer in the wall may be neglected in comparison with the convective heat transfer through the fluid, and the channel may be assumed to have a zero wall thickness.

Due to fabrication considerations and rigidity requirements, the wall thickness of a microchannel is however never negligible compared to its hydraulic diameter (Cole et al., 2014). Hence, heat transfer by conduction in the wall can gof heat conduction through the wall, microscale fluid flow and heat transfer involve many factors that can be significant, such as the non-equilibrium conditions at the fluid-solid wall interface, the shear work, axial conduction, viscous dissipation, pressure work, thermal creep and compressibility effects. Heat transfer in micro-devices is a significant subject for many different engineering applications, such as micro heat exchangers, micro pumps, actuators and sensors. Heat transfer at microscale is dependent on many different parameters that can have significant effects when compared with macro scale problems, therefore investigation of heat transfer phenomena at micro scale is a crucial research area. Conjugate heat transfer in macrochannels was extensively studied in the literature (Shah and London, 1978; Faghri and Sparrow, 1980; Kuo and Lin, 1988) and is still an area of research interest as can be seen in recent articles on the subject. Moreover, convective heat transfer in microchannels with wall thickness effects was investigated in many recent publications, e.g., Darici, et al. (2015); Avci, et al. (2012); Knupp et al. (2013); Kabar et al. (2013), and Ramadan, (2019). Microchannel wall thickness and wall material thermal conductivity effects on heat transfer are among the many factors that are extensively studied in the literature, such as wall thermal diffusivity, the axial conduction in the fluid, rarefaction degree, and Biot number. Analysis of conjugate heat transfer in a parallel-plate microchannel with a heat source at the channel wall is presented in (Cole et al., 2014), considering axial conduction effects in both the fluid and the adjacent wall. Solutions are obtained by the method of Green's functions, and numerical results for the temperature and heat transfer coefficient are obtained for different wall materials, Peclet 
number and Knudsen number. Effects of viscous dissipation, shear work and flow work are however neglected in (Cole et al., 2014). A single domain formulation of conjugated conduction-convection heat transfer problems in parallel plate channel was developed by (Knupp et al., 2012), considering only transverse conduction in the wall. The analysis by (Knupp et al., 2012) was extended to take into account the axial conduction effects at both the wall and fluid regions (Knupp et al., 2013). The results confirm the adequacy of the proposed method to handle conjugated heat transfer in microchannels, when axial conduction effects are accounted for. Effects of other factors such as viscous dissipation, shear work and flow work are howvere, not considered. Using the Generalized Integral Transform Technique, the transient behavior of conjugate heat transfer in microchannel laminar flow was investigated by (Knupp et al., 2015), considering axial conduction and preheating or cooling of the upstream region of the heat exchange section. Nonino et al. (2009) analyzed numerically conjugate heat transfer in a microchannel of circular cross section with an isoflux boundary condition, including the axial heat conduction effects in the pipe wall. The effects of wall thickness and wall material thermal conductivity were investigated. A numerical study of conjugate convective heat transfer of a liquid flow in a circular tube with constant outside wall temperature was performed in (Zhang et al., 2010). The results presented show that heat transfer is most sensitive to the wall-to-fluid thermal conductivity ratio over the range presented. The authors concluded that the wall axial heat conduction leads to a more uniform inner wall surface heat flux. Rahimi and Mehryar (2012) investigated numerically heat transfer of a simultaneously developing laminar flow in a microtube with a constant wall heat flux condition. The effects of the thermal conductivity and wall thickness on the Nusselt number are presented. The results presented therein show that the conduction in the wall decreases the local Nusselt number. Avci et al. (2012) studied numerically conjugate heat transfer in a microtube liquid flow with a constant wall heat flux. Effects of different parameters such as the wall thickness and its thermal properties, channel length and viscous dissipation on Nusselt number, the temperature, wall heat flux at the inner wall side were investigated. It was shown that the heat conduction in the wall reduces the Nusselt number in the entrance region. Conjugate heat transfer of water flow in a partially heated microtube was investigated in (Lelea, 2007), illustrating the influence of the tube wall thickness, tube material and Reynolds number on heat transfer. In a numerical study, Kabar et al. (2013) investigated the effects of rarefaction on heat transfer in a developing flow in parallel plates microchannel with non-zero wall thickness, using an isoflux boundary condition. The effects of different parameters on heat transfer were investigated. A numerical study of the combined effects of viscous dissipation, flow work, shear work and axial diffusion in a gas flow in a microtube is presented in (Ramadan, 2017), with a constant wall heat flux boundary condition, and the results therein demonstrate the significance of these factors on heat transfer in microchannels. The heat conduction in the wall is however neglected. Analysis of conjugated convection - conduction heat transfer in a microtube of finite length with constant wall heat flux boundary condition was presented in (Ramadan, 2019a), taking into account the shear work, axial conduction as well as the combined viscous dissipation and pressure work effects with a constant wall heat flux boundary condition. Ramadan et al. (2019b) investigated numerically a simultaneously developing parallel plate microchannel gas flow and conjugate heat transfer, using the vorticity-stream function formulation. Rarefaction, shear work, viscous dissipation, pressure work, axial conduction, and conjugate effects on heat transfer characteristics are investigated. Sen and Darici (2017) investigated numerically transient conjugate heat transfer of a hydrodynamically developed and thermally developing laminar flow in a circular microchannel, considering the effects of rarefaction, viscous dissipation and axial conduction. Effect of shear work and pressure work were not considered. The problem was shown to depend on the Peclet number, the Knudsen number, the Brinkman number and the wall thickness ratio. Ayden and Avci (2006) investigated laminar forced convective heat transfer of a Newtonian fluid in a micropipe is analyzed by taking the viscous dissipation effect, the velocity slip and the temperature jump at the wall into account. The flow was assumed to be hydrodynamically and thermally fully developed, and axial conduction, pressure work and shear work effect were neglected. An analytical solution of the energy equation for a hydrodynamically fully developed, thermally developing flow in a microtube was performed by Barisik et al. (2015) using the Gram-Schmidt orthogonalization technique. The model presented includes axial conduction, viscous dissipation and rarefaction effects, but neglecting the shear work and pressure work effects.

The present analysis combines the effects of viscous dissipation, pressure work, shear work and axial conduction in the gas, together with heat conduction in the microtube wall in both the axial and radial directions, with a constant wall temperature boundary condition. For this case, the thermally fully developed conditions at the tube exit are attainable if the tube is long enough, and the outflow thermal boundary condition is well defined. The current study therefore, reveals the influence of different factors on the thermal entrance length in a microtube gas flow with a constant outer 
wall temperature.

\section{Problem formulation and numerical solution method}

The problem geometry is as shown in Fig. 1. The gas flow through the microtube is assumed to be hydrodynamically fully developed. The flow is assumed steady, laminar, and incompressible with constant fluid properties. The gas enters the microtube with a constant inlet temperature, $T_{o}$ (Fig. 1). The energy equations for the fluid and the solid wall are:

$$
\begin{aligned}
& u \frac{\partial T_{f}}{\partial z}=\alpha\left[\frac{1}{r} \frac{\partial}{\partial r}\left(r \frac{\partial T_{f}}{\partial r}\right)+\frac{\partial^{2} T_{f}}{\partial z^{2}}\right]+\frac{\mu}{\rho c_{p}}\left(\frac{\partial u}{\partial r}\right)^{2}+\frac{u}{\rho c_{p}} \frac{d P}{d z}, \quad 0 \leq r \leq R_{i} \\
& \frac{1}{r} \frac{\partial}{\partial r}\left(r \frac{\partial T_{w}}{\partial r}\right)+\frac{\partial^{2} T_{w}}{\partial z^{2}}=0, \quad R_{i} \leq r \leq R_{o}
\end{aligned}
$$

where $u$ is the velocity profile given by Eq. (3):

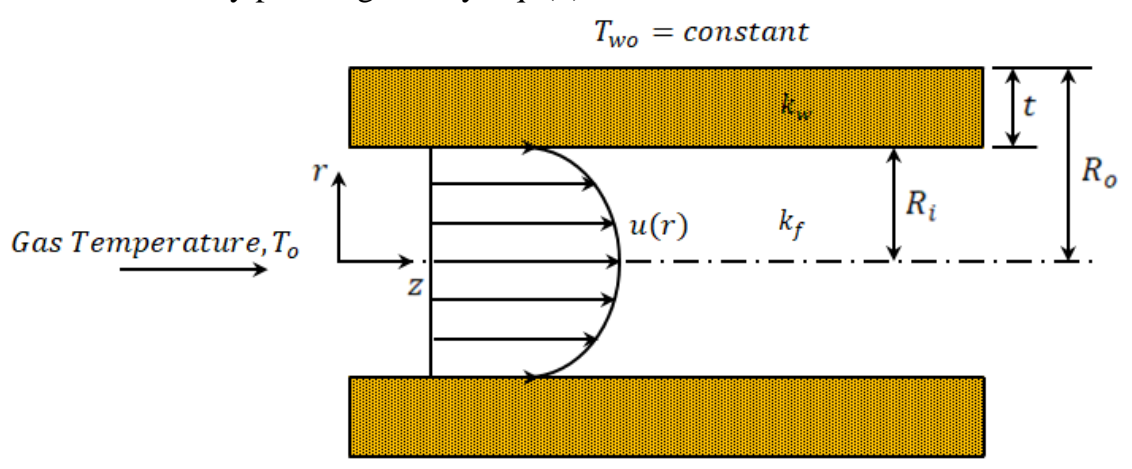

Fig. 1 Schematic of the Problem geometry

$$
u(r)=\frac{2 u_{m}}{1+8 \beta_{V} K n}\left[1+4 \beta_{V} K n-\left(r / R_{i}\right)^{2}\right]
$$

The Knudsen number $(K n)$ in Eq. (3) is defined as:

$$
K n=\frac{\lambda}{D_{i}}=\frac{\lambda}{2 R_{i}}
$$

and $\beta_{V}$ is a parameter related to the momentum accommodation coefficient $\sigma_{V}$ as:

$$
\beta_{V}=\frac{2-\sigma_{V}}{\sigma_{V}}
$$

The boundary conditions for the energy equations are taken as follows:

$$
\begin{aligned}
& T_{w}\left(R_{o}, z\right)=T_{w o}=\text { Constant } \\
& T_{f}(r, 0)=T_{o}, 0 \leq r \leq R_{i}
\end{aligned}
$$

Different boundary conditions can be taken at the tube wall inlet (i.e., at $z=0, R_{i} \leq r \leq R_{o}$ ); adiabatic wall, constant temperature at $T_{o}$, constant temperature at $T_{w o}$ or a linear variation of the wall temperature between $T_{o}$ and $T_{w o}$. In this analysis, and to eliminate the end effects on heat transfer, the tube wall at both ends is taken as insulated:

$$
\left(\frac{\partial T_{w}}{\partial z}\right)_{z=0}=\left(\frac{\partial T_{w}}{\partial z}\right)_{z \rightarrow \infty}=0, \quad R_{i} \leq r \leq R_{o}
$$

The symmetry and the fully developed flow boundary conditions are given respectively as:

$$
\begin{aligned}
& \left(\frac{\partial T_{f}}{\partial r}\right)_{r=0}=0, \quad 0 \leq z \leq L \\
& \left(\frac{\partial T_{f}}{\partial z}\right)_{z \rightarrow \infty}=0
\end{aligned}
$$

The shear work contribution to the heat flux at the gas - solid interface can be significant at high Knudsen number. The boundary condition at the interface, at the considering the shear work contribution is: 


$$
k_{w}\left(\frac{\partial T_{w}}{\partial r}\right)_{r=R_{i}}=k_{f}\left(\frac{\partial T_{f}}{\partial r}\right)_{r=R_{i}}+\mu\left(u \frac{\partial u}{\partial r}\right)_{r=R_{i}}
$$

and the temperature jump condition at the fluid - solid wall interface is given as:

$$
T_{w}\left(z, R_{i}\right)=T_{f}\left(z, R_{i}\right)+\frac{2-\sigma_{T}}{\sigma_{T}} \frac{2 \gamma}{\gamma+1} \frac{\lambda}{P r}\left(\frac{\partial T_{f}}{\partial r}\right)_{r=R_{i}}
$$

where the parameters, $\sigma_{T}, \gamma, \lambda, \operatorname{Pr}$ in Eq. (12) are respectively, the thermal accommodation coefficient, specific heat ratio, mean free path and Prandtl number.

Using the dimensionless variables:

$$
\xi=\frac{z}{R_{i}}, \quad \eta=\frac{r}{R_{i}}, \quad \theta(\xi, \eta)=\frac{T-T_{w o}}{T_{o}-T_{w o}},
$$

Equations (1) to (3) are re-written as:

$$
\begin{aligned}
& \frac{\left(C_{2}-\eta^{2}\right) P e}{C_{3}} \frac{\partial \theta_{f}}{\partial \xi}=\frac{1}{\eta} \frac{\partial \theta_{f}}{\partial \eta}+\frac{\partial^{2} \theta_{f}}{\partial \eta^{2}}+\frac{\partial^{2} \theta_{f}}{\partial \xi^{2}}+\frac{16 B r}{C_{3}^{2}} \eta^{2}-\frac{16 B r}{C_{3}^{2}}\left(C_{2}-\eta^{2}\right) \\
& \frac{\partial^{2} \theta_{w}}{\partial \eta^{2}}+\frac{1}{\eta} \frac{\partial \theta_{w}}{\partial \eta}+\frac{\partial^{2} \theta_{w}}{\partial \xi^{2}}=0, \quad 1 \leq \eta \leq 1+b \\
& u(\eta)=\frac{2 u_{m}}{1+8 C_{1}}\left(C_{2}-\eta^{2}\right)
\end{aligned}
$$

where $b$ is the ratio of the tube wall thickness to its inner radius $\left(b=t / R_{i}\right)$, and $u_{m}$ is the mean velocity. The Brinkman number, Peclet number and the constants $C_{1}, C_{2}, C_{3}$ in Eqs. (14) and (16) are defined as:

$$
\begin{aligned}
B r & =\frac{\mu u_{m}^{2}}{k_{f}\left(T_{o}-T_{w o}\right)} \\
P e & =\frac{2 u_{m} R_{i}}{\alpha} \\
C_{1} & =\beta_{V} K n, \quad C_{2}=1+4 C_{1}, \quad C_{3}=1+8 C_{1}
\end{aligned}
$$

The dimensionless boundary conditions take the form:

$$
\begin{aligned}
& \theta_{w}(\xi, 1+b)=0.0 \\
& \theta_{f}(0, \eta)=1.0 \\
& \left(\frac{\partial \theta_{w}}{\partial \xi}\right)_{\xi=0}=\left(\frac{\partial \theta_{w}}{\partial \xi}\right)_{\xi \rightarrow \infty}=0 \\
& \left(\frac{\partial \theta_{f}}{\partial \eta}\right)_{\eta=0}=0 \\
& \left(\frac{\partial \theta_{f}}{\partial \xi}\right)_{\xi \rightarrow \infty}=0 \\
& \frac{k_{w}}{k_{f}}\left(\frac{\partial \theta_{w}}{\partial \eta}\right)_{\eta=1}=\left(\frac{\partial \theta_{f}}{\partial \eta}\right)_{\eta=1}-\frac{32 C_{1} B r}{C_{3}^{2}}
\end{aligned}
$$

The temperature jump condition (Eq. 12) is:

$$
\theta_{f}(\xi, 1)+2 \beta_{T}^{*}\left(\frac{\partial \theta_{f}}{\partial \eta}\right)_{\eta=1}=\theta_{w}(\xi, 1)
$$

The constant $\beta_{T}^{*}$ in Eq. (26) is:

$$
\beta_{T}^{*}=\frac{2 \gamma}{\gamma+1} \frac{K n}{P r} \beta_{T}
$$

and the parameter $\beta_{T}$ is related to the thermal accommodation coefficient $\sigma_{T}$ as:

$$
\beta_{T}=\frac{2-\sigma_{T}}{\sigma_{T}}
$$


The wall heat flux at the solid-gas interface due to conduction only, is given as:

$$
q_{c}=k_{f}\left(\frac{\partial T_{f}}{\partial r}\right)_{r=R_{i}}
$$

Using Eq. (13) in Eq. (29), the conductive wall heat flux at the inner wall side in dimensionless form is:

$$
Q_{c}=\left(\frac{\partial \theta_{f}}{\partial \eta}\right)_{\eta=1}=\frac{k_{w}}{k_{f}}\left(\frac{\partial \theta_{w}}{\partial \eta}\right)_{\eta=1}=\frac{q_{c} R_{i}}{k_{f}\left(T_{o}-T_{w o}\right)}
$$

The total heat flux at the wall due to both heat conduction and shear work, $q_{s w}$ is:

$$
q_{w i}=q_{c}-q_{s w}=k_{f}\left(\frac{\partial T_{f}}{\partial r}\right)_{r=R_{i}}+\mu\left(u \frac{\partial u}{\partial r}\right)_{r=R_{i}}
$$

and the total wall heat flux in dimensionless form is evaluated from Eq. (31) using Eqs. (13) and (16):

$$
Q_{w i}=\left(\frac{\partial \theta_{f}}{\partial \eta}\right)_{\eta=1}-\frac{32 C_{1} B r}{C_{3}^{2}}=\frac{q_{w i} R_{i}}{k_{f}\left(T_{o}-T_{w o}\right)}
$$

The Nusselt number due to heat conduction only, $N u_{t h}$ (i.e., neglecting the shear work) is given as:

$$
N u_{t h}=\frac{q_{c} D_{i}}{k_{f}\left(T_{w i}-T_{m}\right)}
$$

and using Eq. (30) in Eq. (33):

$$
N u_{t h}=\frac{2}{\theta_{w i}-\theta_{m}}\left(\frac{\theta_{f}}{\partial \eta}\right)_{\eta=1}
$$

The Nusselt number taking the shear work contribution into account, $N u_{\text {tot }}$, ( i.e., the heat conduction and the boundary shear work), is written as:

$$
N u_{t o t}=\frac{q_{w i} D_{i}}{k_{f}\left(T_{w i}-T_{m}\right)}=\frac{2}{\theta_{w i}-\theta_{m}}\left[\left(\frac{\partial \theta_{f}}{\partial \eta}\right)_{\eta=1}-\frac{32 C_{1} B r}{C_{3}^{2}}\right]
$$

An analytical expression for the fully developed temperature profile with non-zero Brinkman number can be found by integrating the energy equation and applying the fully developed conditions:

$$
\theta_{f d}(\eta)=\frac{2 B r}{C_{3}^{2}}\left(2 C_{2} \eta^{2}-\eta^{4}-32 C_{1} \beta_{T}^{*}-C_{3}\right)
$$

Using Eq. (36), the fully developed fluid mean temperature $\theta_{m, f d}$, Nusselt number due to conduction $N u_{f d, t h}$ and the total Nusselt number $N u_{f d, t o t}$ are:

$$
\begin{aligned}
& \theta_{m, f d}=\frac{2 B r}{C_{3}^{3}}\left(1-4 C_{2}+4 C_{2}^{2}-2 C_{3}^{2}-64 C_{1} C_{3} \beta_{T}^{*}\right) \\
& N u_{f d, t h}=\frac{64 C_{1} C_{3}}{2 C_{3}^{2}+64 C_{1} C_{3} \beta_{T}^{*}-4 C_{2}^{2}+4 C_{2}-1} \\
& N u_{f d, t o t}=0
\end{aligned}
$$

The fully developed temperature distribution and the mean temperature are clearly dependent on the Brinkman number, while the Nusselt number is not. The sum of the shear work and the diffusive heat flux at the wall is zero, leading to $N u_{f d, t o t}=0$.

\section{Numerical solution method}

A coordinate transformation in the axial direction is implemented to capture the boundary condition far downstream the tube exactly, using the transformation (Ramadan, 2017):

$$
\xi^{*}=1-\frac{1}{1+a \xi}
$$

Where $a$ is a stretching parameter $(a>0)$.

Using Eq. (40), Eq. (14) takes the form: 


$$
F\left(\xi^{*}, \eta\right) \frac{\partial \theta_{f}}{\partial \xi^{*}}=G\left(\xi^{*}\right) \frac{\partial^{2} \theta_{f}}{\partial \xi^{* 2}}+\frac{1}{\eta} \frac{\partial \theta_{f}}{\partial \eta}+\frac{\partial^{2} \theta_{f}}{\partial \eta^{2}}+\frac{16 B r}{C_{3}^{2}} \eta^{2}-\frac{16 B r}{C_{3}^{2}}\left(C_{2}-\eta^{2}\right)
$$

where $F\left(\xi^{*}, \eta\right)$ and $G\left(\xi^{*}\right)$ are given as:

$$
\begin{aligned}
& F\left(\xi^{*}, \eta\right)=\frac{a P e}{C_{3}}\left(C_{2}-\eta^{2}\right)\left(1-\xi^{*}\right)^{2}+2 a^{2}\left(1-\xi^{*}\right)^{3} \\
& G\left(\xi^{*}\right)=a^{2}\left(1-\xi^{*}\right)^{4}
\end{aligned}
$$

In order to avoid the singularity at $\eta=0$, Eq. (41) is expanded at $\eta=0$ in a Maclaurin series. This leads to the following form of the energy equation at the centerline $(\eta=0)$ :

$$
F\left(\xi^{*}, \eta\right) \frac{\partial \theta_{f}}{\partial \xi^{*}}=G\left(\xi^{*}\right) \frac{\partial^{2} \theta_{f}}{\partial \xi^{* 2}}+2 \frac{\partial^{2} \theta_{f}}{\partial \eta^{2}}
$$

The solid wall energy equation (Eq. 15) becomes:

$$
G\left(\xi^{*}\right) \frac{\partial^{2} \theta_{w}}{\partial \xi^{* 2}}-H\left(\xi^{*}\right) \frac{\partial \theta_{w}}{\partial \xi^{*}}+\frac{\partial^{2} \theta_{w}}{\partial \eta^{2}}+\frac{1}{\eta} \frac{\partial \theta_{w}}{\partial \eta}=0
$$

where $H\left(\xi^{*}\right)$ is:

$$
H\left(\xi^{*}\right)=2 a\left(1-\xi^{*}\right)^{3}
$$

And the boundary condition (Eq. 24) becomes:

$$
\left(\frac{\partial \theta_{w}}{\partial \xi^{*}}\right)_{\xi^{*}=1}=\left(\frac{\partial \theta_{f}}{\partial \xi^{*}}\right)_{\xi^{*}=1}=0
$$

Using finite differences, a computer code is developed to solve the energy equations of the fluid and the solid wall utilizing the iterative line Gauss-Seidel iterative technique. Second order accurate central differences are used to approximate all the derivatives in Eqs. $(37,40,41)$ except for the advective term in Eq. (41), where a second order backward difference formula is used. All the results presented in this work are performed with $\gamma=1.4, \operatorname{Pr}=0.7$. Without loss of generality, the accommodation coefficients are taken as $\sigma_{T}=\sigma_{V}=1$ throughout this work. A grid sensitivity analysis is performed to ensure a grid independent solution is obtained. This is shown in Fig. 2 with different grid sizes and a stretching factor $a=0.2$. A grid independent solution is obtained with $\Delta \xi^{*}=0.001, \Delta \eta=0.005$. The grid size used in all of the results presented in this work is $\Delta \xi^{*}=0.0005, \Delta \eta=0.0025$ with a convergence criterion of $\left|\theta^{n+1}-\theta^{n}\right| \leq 10^{-6}$, where $\theta^{n}, \theta^{n+1}$ are the solutions at two successive iterations.
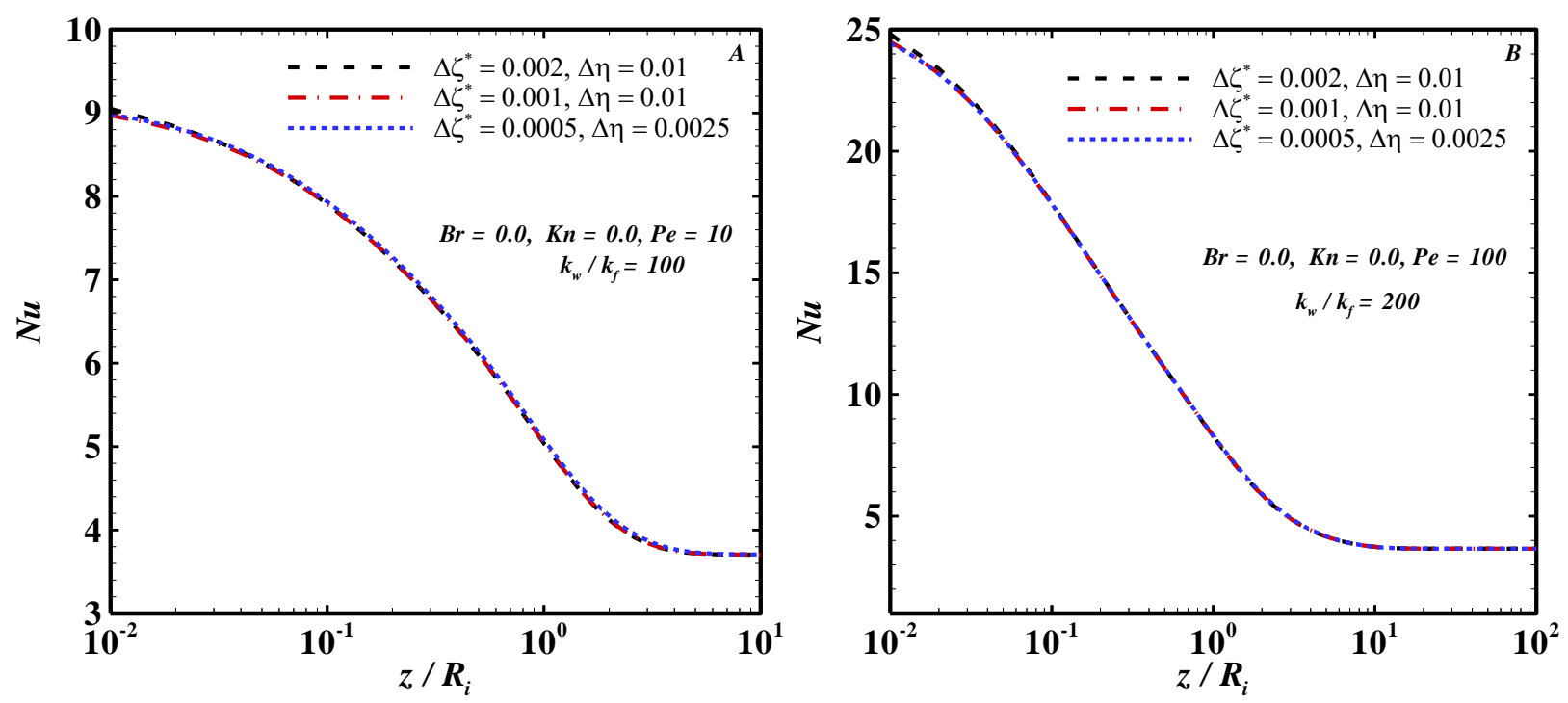

Fig. 2 Code verification: Numerical solutions with different grid sizes and grid independent solutions for (A) $P e=10$, $k_{w} / k_{f}=100$ and (B) $P e=100, . k_{w} / k_{f}=200$. 


\section{Results and discussion}

The effect of heat conduction in the tube wall on heat convection is presented in this section in terms of the tube wall thickness and the wall to fluid thermal conductivity ratio, taking into consideration the viscous dissipation, pressure work, shear work and axial conduction effects. The numerical solution is verified via comparison of the current results with well-established data in the literature for special cases of the model in this study. Figure 3 shows the current solution of the local Nusselt number and data extracted from Shmidt and Zeldin (1970) with $P e=1$ (Fig.3-A) and from Jeong and Jeong (2006) with $P e=100$ (Fig. 3-B). The present solution clearly compares well with the other two solutions in the thermal entrance region with $B r=0$ (both viscous dissipation and pressure work are zero) and the fully developed Nusselt number approaches the well-established result, i.e., $N u_{f d} \approx 3.66$. As both the pressure work and viscous dissipation are included in the present model, a second case with a non-zero Brinkman number $\left(\mathrm{Br}=-10^{-4}\right)$ is shown in Figure 3. With this small Brinkman number, the solution should be necessarily very close to the case with $\mathrm{Br}=0$ in the developing region, where the thermal entrance effect dominates by far the combined effect of viscous dissipation and flow work. As the thermal entrance effect continuously decreases downstream, the combined effect of viscous dissipation and pressure work becomes increasingly more dominant and results in a zero fully developed Nusselt number as explained in (Ramadan, 2017).
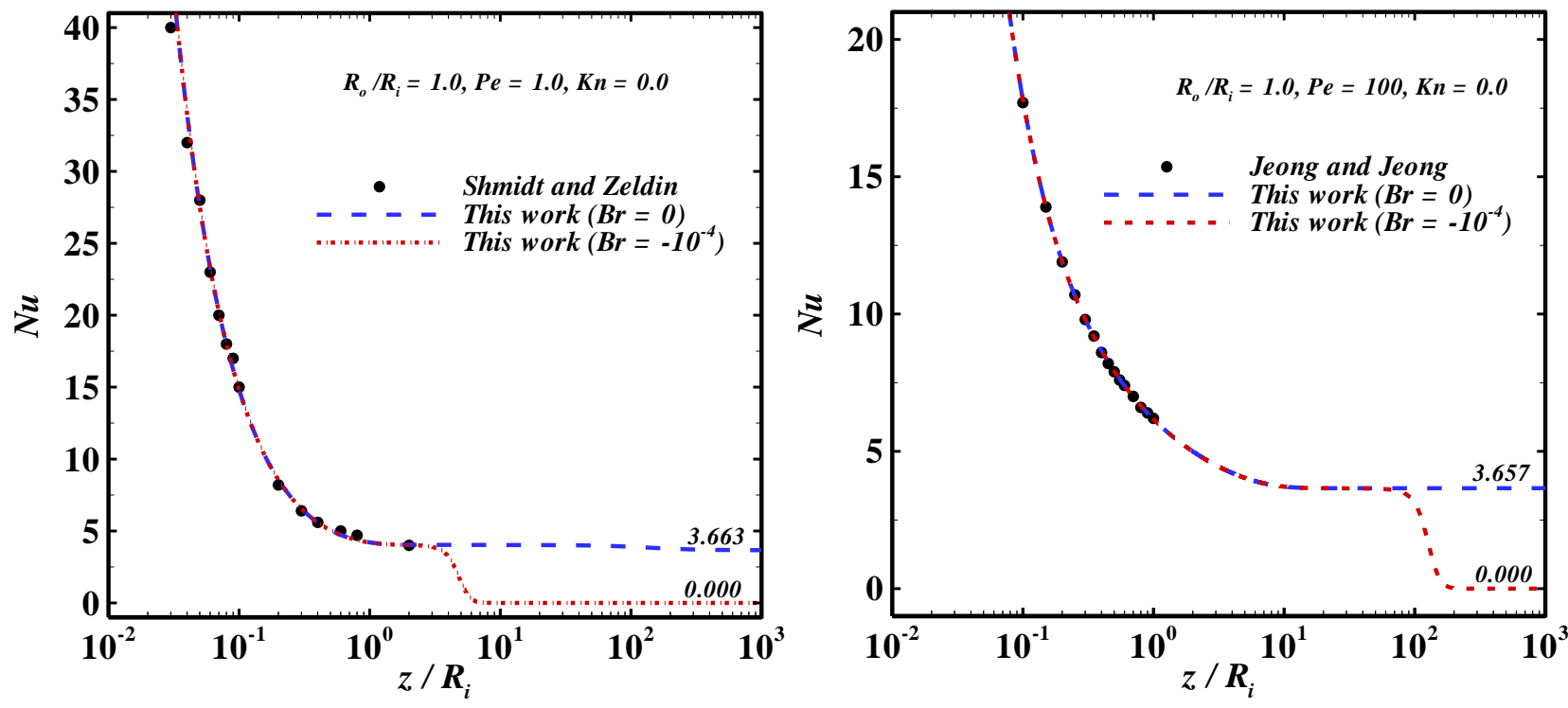

Fig. 3 Variation of the local Nusselt number along the tube: A comparison between current results with data from the literature with zero pipe wall thickness and no rarefaction at (A) $P e=1.0$ and (B) $P e=100$

The temperature profiles obtained numerically are also compared with that derived analytically in this work (Eq. 36). This is shown in Fig. 4 for different flow parameter, with $k_{w} / k_{f}=10$ (Fig. $4-\mathrm{A}$ ) and $k_{w} / k_{f}=200$ (Fig. 4-B). The numerical and analytical temperature profiles compare very well, as can be seen in the Figure. Moreover, the numerical and analytical solutions of the fully developed Nusselt number with $B r \neq 0$ and the mean fluid temperature $\theta_{m, f d}$ for $B r=-0.1$ are compared and the results are displayed in Table $1\left(\theta_{m, f d}\right.$ is dependent on $B r$, while $N u_{t h}$ is not, as is clear from Eqs. 37-39. The numerical and analytical results clearly compare very well, with an error of less than $0.1 \%$. 

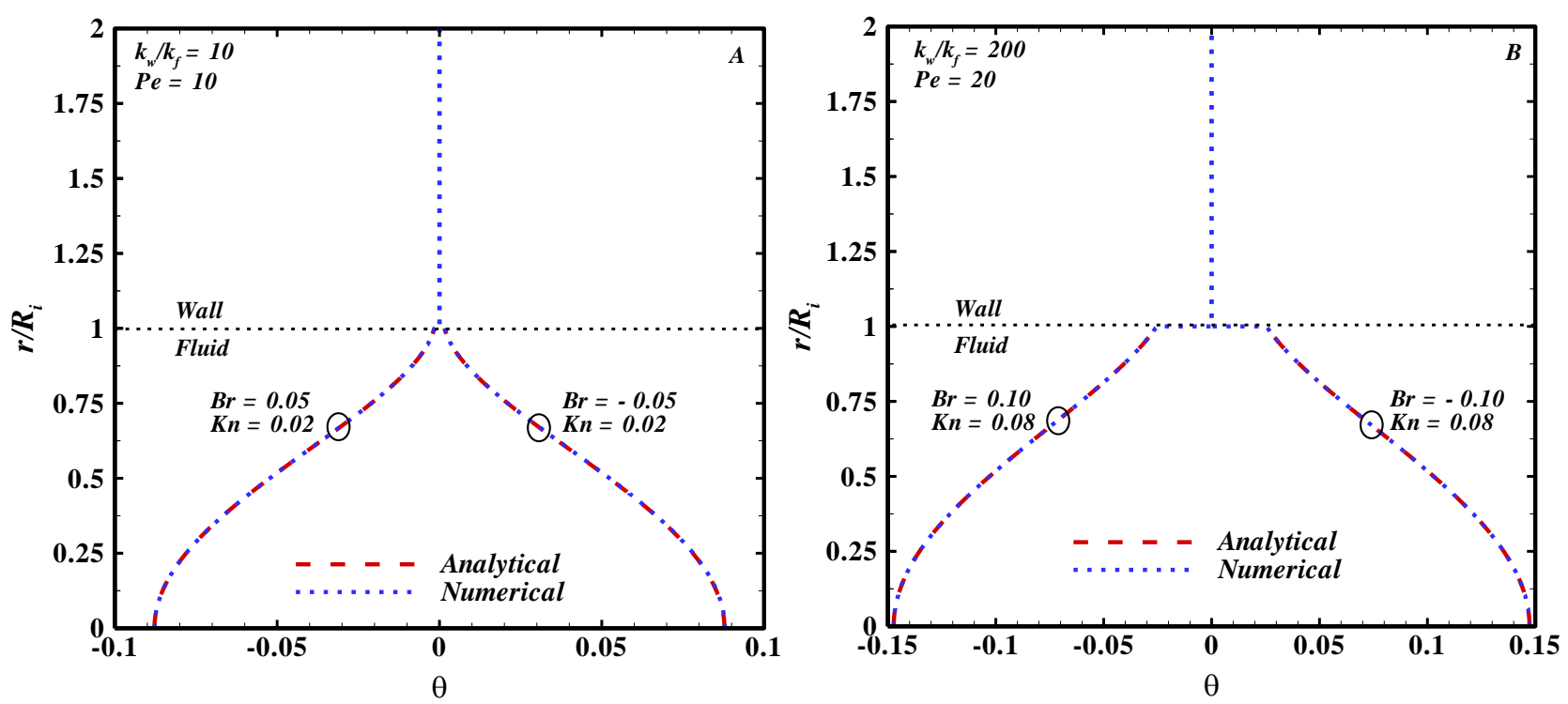

Fig. 4 A comparison between the current numerical and analytical solutions of the fully developed temperature profiles with $k_{w} / k_{f}=10, \quad(\mathrm{~A})$ and $k_{w} / k_{f}=200(\mathrm{~B})$.

Table 1 A Comparison between the Exact and Numerical Results: Fully Developed Values of Nusselt number for any $\mathrm{Br} \neq 0$ and the mean gas temperature (with $\mathrm{Br}=-0.1$ )

\begin{tabular}{|c|c|c|c|c|c|c|}
\hline \multirow{2}{*}{$K n$} & \multicolumn{2}{|c|}{$N u_{t h}$} & \multicolumn{2}{c|}{$N u_{\text {tot }}$} & \multicolumn{2}{c|}{$\theta_{m}(B r=-0.1)$} \\
\cline { 2 - 7 } & Exact & Numerical & Exact & Numerical & Exact & Numerical \\
\hline 0.0 & 0.0000 & 0.0011 & 0 & 0.0011 & 0.1000 & 0.1000 \\
\hline 0.02 & 1.0643 & 1.0652 & 0 & 0.0009 & 0.0894 & 0.0894 \\
\hline 0.04 & 1.7174 & 1.7181 & 0 & 0.0007 & 0.0856 & 0.0856 \\
\hline 0.06 & 2.0601 & 2.0607 & 0 & 0.0006 & 0.0851 & 0.0851 \\
\hline 0.08 & 2.2044 & 2.2048 & 0 & 0.0005 & 0.0864 & 0.0864 \\
\hline 0.10 & 2.2326 & 2.2330 & 0 & 0.0004 & 0.0885 & 0.0885 \\
\hline
\end{tabular}

The effects of the wall thickness of the tube on the Nusselt number, mean temperature, inner wall temperature and the inner wall heat flux are shown in Fig. 5 with no rarefaction $(K n=0)$, also with no shear work and no viscous dissipation or flow work and, hence $N u_{t o t}=N u_{t h}$. The case with $R_{o} / R_{i}=1$ (i.e., zero wall thickness) is also included. Obviously, as the wall thickness decreases, the solutions approach that corresponding to the zero wall thickness case. This is also an evidence of the correctness of the numerical solution procedure and results. For the parameters shown in the figure, the wall thickness has a slight effect on the local Nusselt number in the entrance region, where the tube is taken as insulted at both ends. Moreover, Fig. 5 shows that the thermal entrance length also increases with increasing the wall thickness, with less uniform temperature along the inner wall, and lower inner wall heat flux (in absolute sense) along the tube. The trend shown in Fig. 5 is due to the fact that the resistance to heat conduction in the wall increases with the wall thickness. 

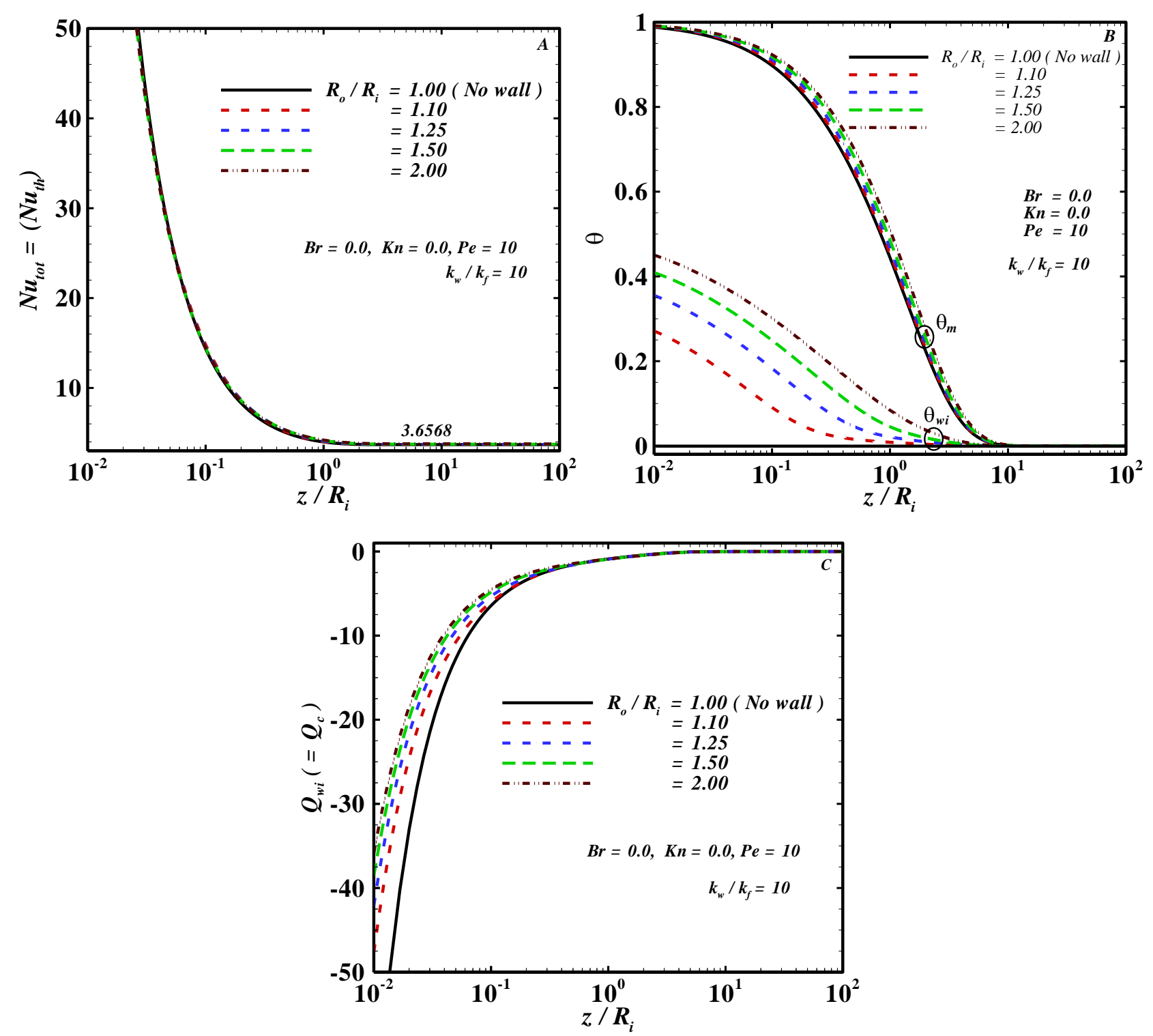

Fig. 5 Tube wall thickness effect on the local Nusselt number (A), mean and inner wall temperatures (B), and inner wall heat flux (C) with no rarefaction, viscous dissipation or flow work.

The microtube wall thickness effect on $N u_{t h}, N u_{t 0 t}, \theta_{w i}, \theta_{m}$ and $Q_{w i}$ with rarefaction $(K n=0.02)$ is presented in Fig. 6 for $\mathrm{Br}<0$ (gas heating) and in Fig. 7 for cooling $(B r>0)$, with $k_{w} / k_{f}=200$. With high wall thermal conductivity (relative to that of the gas), the wall resistance to conduction becomes negligibly small, and with insulated tube ends, the heat flux through the wall remains almost the same and is considerably close to the case with zero wall thickness. Hence for $k_{w} \gg k_{f}$ and with insulated tube ends, the wall thickness has negligible effects on heat transfer characteristics, as Figs. 6 and 7 clarify. The fully developed Nusselt number due to conduction only is $N u_{t h}=1.0652$, while that considering both conduction and shear work is $N u_{t o t}=0.0009$, in excellent agreement with the analytical solution obtained in this work (Table 1). The total wall heat flux in the fully developed region is also zero as is clear in these results. Therefore, the length of a microtube for efficient heat exchange between the gas and the tube wall may not need to exceed the thermal entrance length. A shorter tube also leads to less frictional losses. 

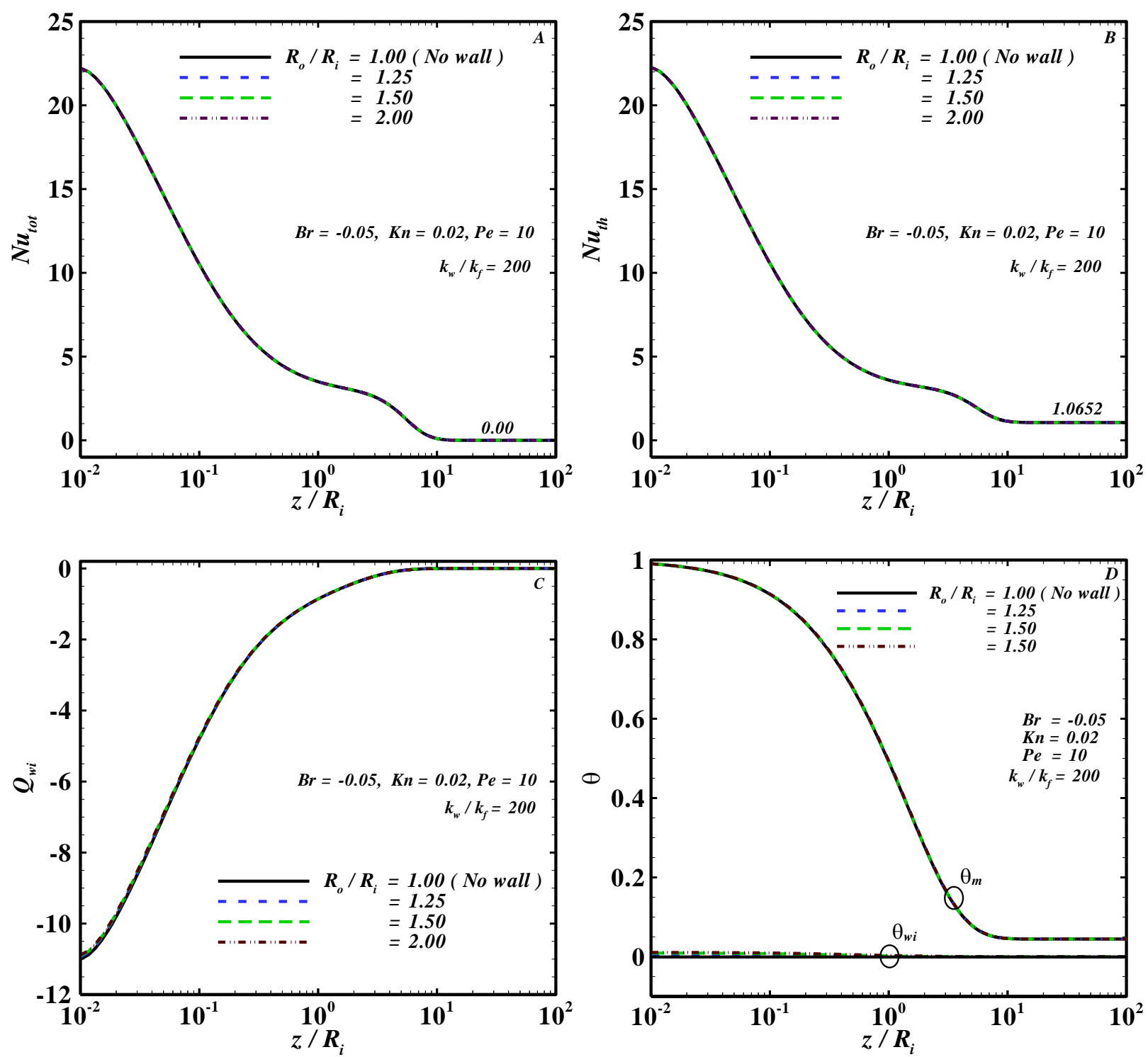

Fig. 6 Tube wall thickness effect on the local Nusselt number (A, B), the inner wall heat flux (C) and the corresponding mean and inner wall temperatures (D), with $\mathrm{Br}=-0.05, \mathrm{Kn}=0.02, \mathrm{Pe}=10$. 

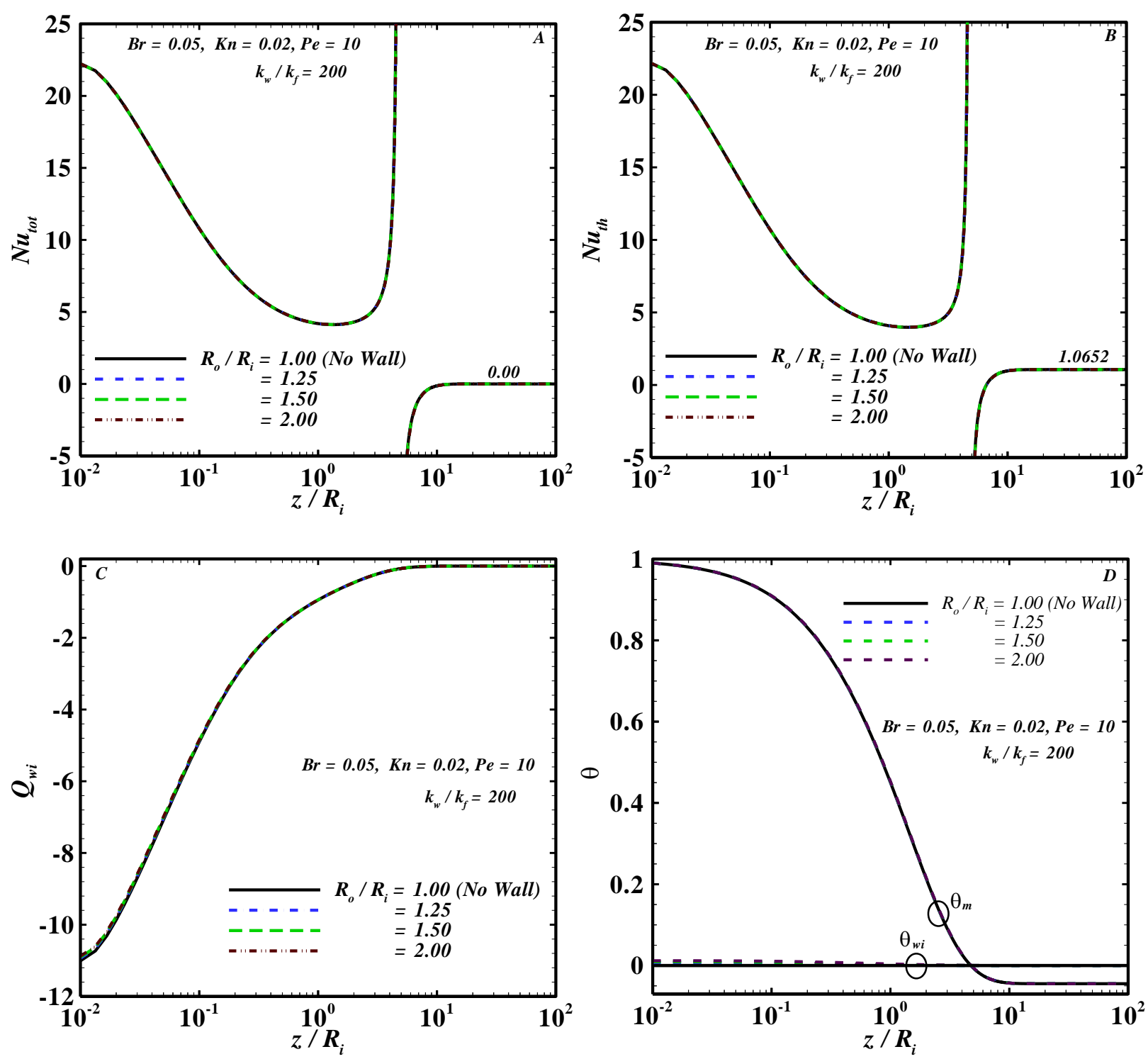

Fig. 7 Tube wall thickness effect on the local Nusselt number (A, B), the inner wall heat flux (C) and the corresponding mean and inner wall temperatures (D), with $\mathrm{Br}=0.05, \mathrm{Kn}=0.02, \mathrm{Pe}=10$.

Figure 8 displays the wall-to fluid thermal conductivity ratio $\left(k_{w} / k_{f}\right)$ effect on Nusselt number, inner wall heat flux as well as the fluid mean temperature and inner wall temperature. The smaller the thermal conductivity ratio the more pronounced is the effect on the thermal entrance length, the Nusselt number and the other heat transfer parameters shown in the figure. Smaller $k_{w} / k_{f}$ leads to higher thermal entrance length and relatively a more uniform inner wall heat flux along the tube wall. Figure 8 also shows that for a fixed tube wall thickness, the heat transfer parameters are almost the same when $k_{w} \gg k_{f}$, where the wall thermal resistance becomes negligibly small.

The effect of the Peclet number on Nusselt number and the inner wall heat flux is presented in Fig. 9 for both zero and non-zero wall thickness. The thermal entrance length clearly increases with increasing the Peclet number, and doubling the Peclet number almost doubles the entrance length. The inner wall heat flux increases with the Peclet number. These two observations are due to the fact that higher Peclet number leads to lower axial conduction, hence more heat transfer in the radial direction. The lower inner wall heat flux with the non-zero tube wall thickness at the same Peclet number is also seen in Fig. 5. Moreover, the higher the Peclet number (i.e., lower axial conduction) the higher the Nusselt number for both the zero and the non-zero wall thicknesses. Figure 10 displays the rarefaction effect on the Nusselt number and the corresponding inner wall heat flux with zero and non-zero wall thickness, for $k_{w} / k_{f}=100$. The Nusselt number and the inner wall heat flux in the thermal entrance region are shown to decrease significantly with increasing rarefaction. The thermal entrance length extends longer with increasing rarefaction. Wall 
heat conduction for the case presented in Fig. 10 has only a slight effect on heat transfer parameters, since the wall thermal conductivity is much higher than that of the gas, hence low wall thermal resistance to conduction.
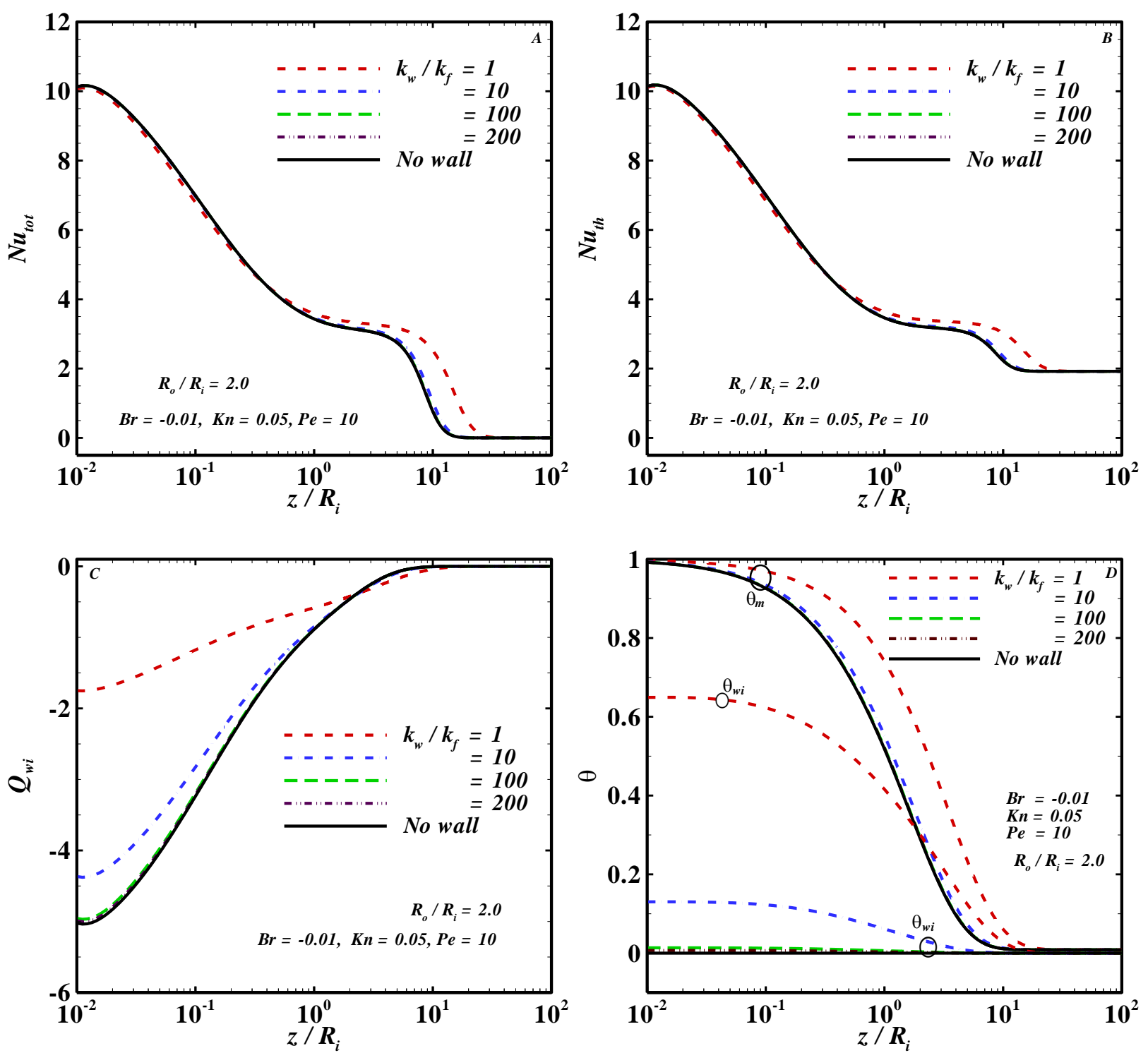

Fig. 8 Tube wall thermal conductivity effect on the local Nusselt number (A, B), the inner wall heat flux (C) and the corresponding mean and inner wall temperatures (D), with $\mathrm{Br}=-0.01, \mathrm{Kn}=0.05, \mathrm{Pe}=10$. 

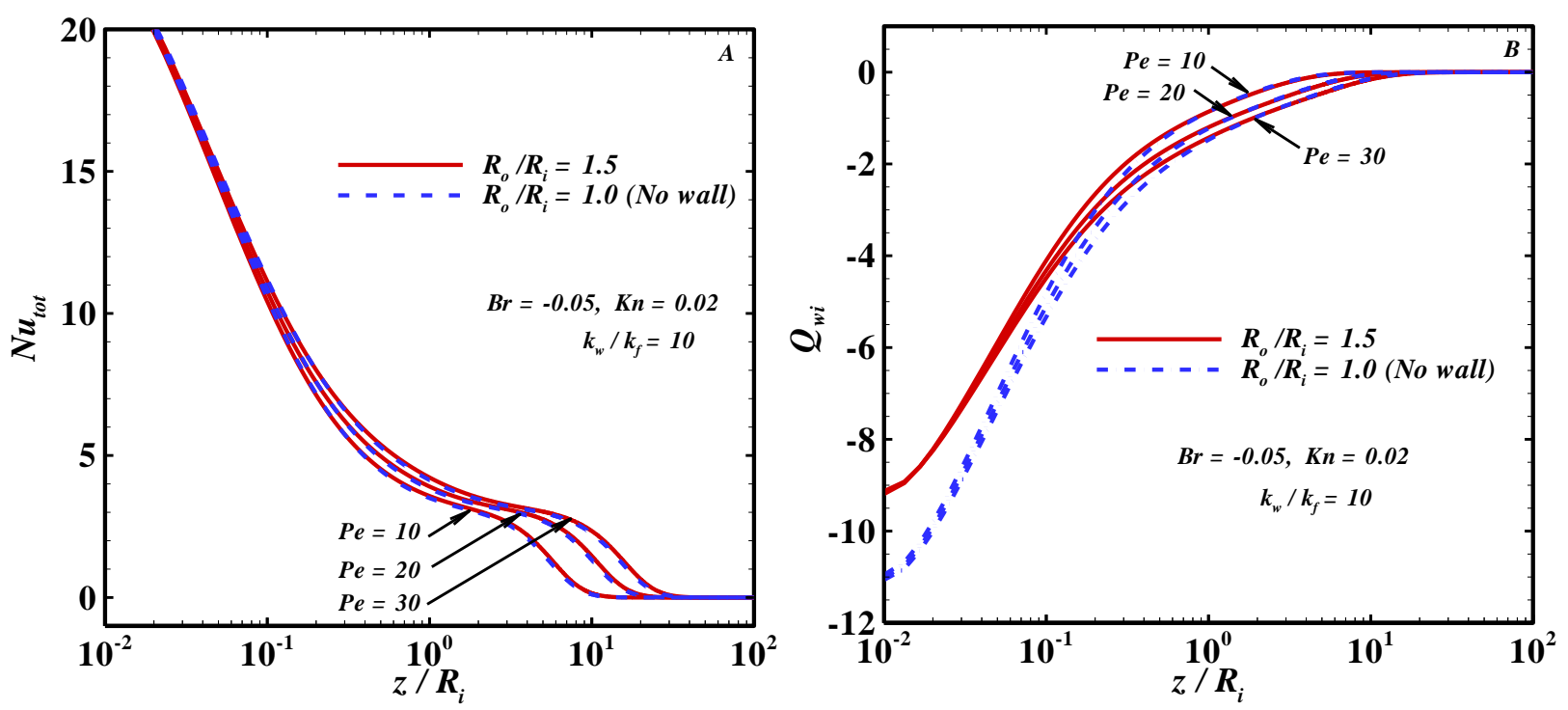

Fig. 9 Variation of the local Nusselt number and inner wall heat flux for different values of Peclet number, with and without a wall.
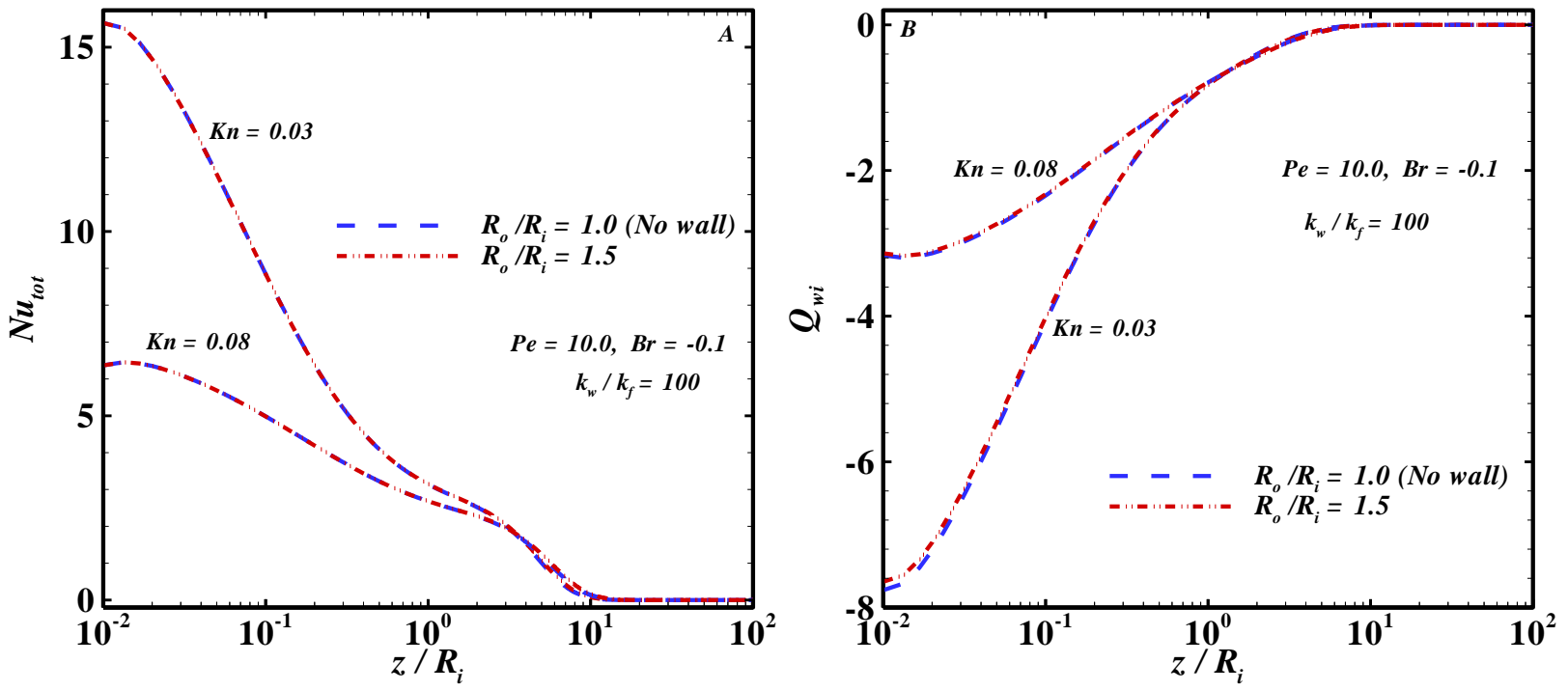

Fig. 10 Effect of rarefaction on the local Nusselt number (A) and inner wall heat flux (B), with and without a wall.

The temperature distribution in the microtube is presented in Figs. 11 and 12 for both gas heating (A) and cooling (B). The gas temperature changes most rapidly at the beginning of the entrance region where the temperature difference between the wall and the gas is the highest. For the gas heating case $(B r<0)$, the dimensionless temperature never drops below zero (i.e., $T<T_{w o}$ ), as is clear in Fig. 11 (A) and Fig. 12(A). While for the gas cooling case $(B r>0)$, the dimensionless temperature drops below zero in the thermally fully region (i.e., $T>T_{w o}$ ), where in this case both the thermal entrance effect and the shear work effect contribute to energy loss from the gas. The temperature distribution in the wall is much more uniformly distributed due to the high thermal conductivity of the wall material to that of the gas. The temperature jump at the wall (i.e., the temperature difference between the inner wall temperature and the gas temperature at the wall), is clearly seen in both Figs. 11 and 12. The temperature jump at the wall decreases continuously downstream, following the decrease in the gas temperature gradient at the wall. 


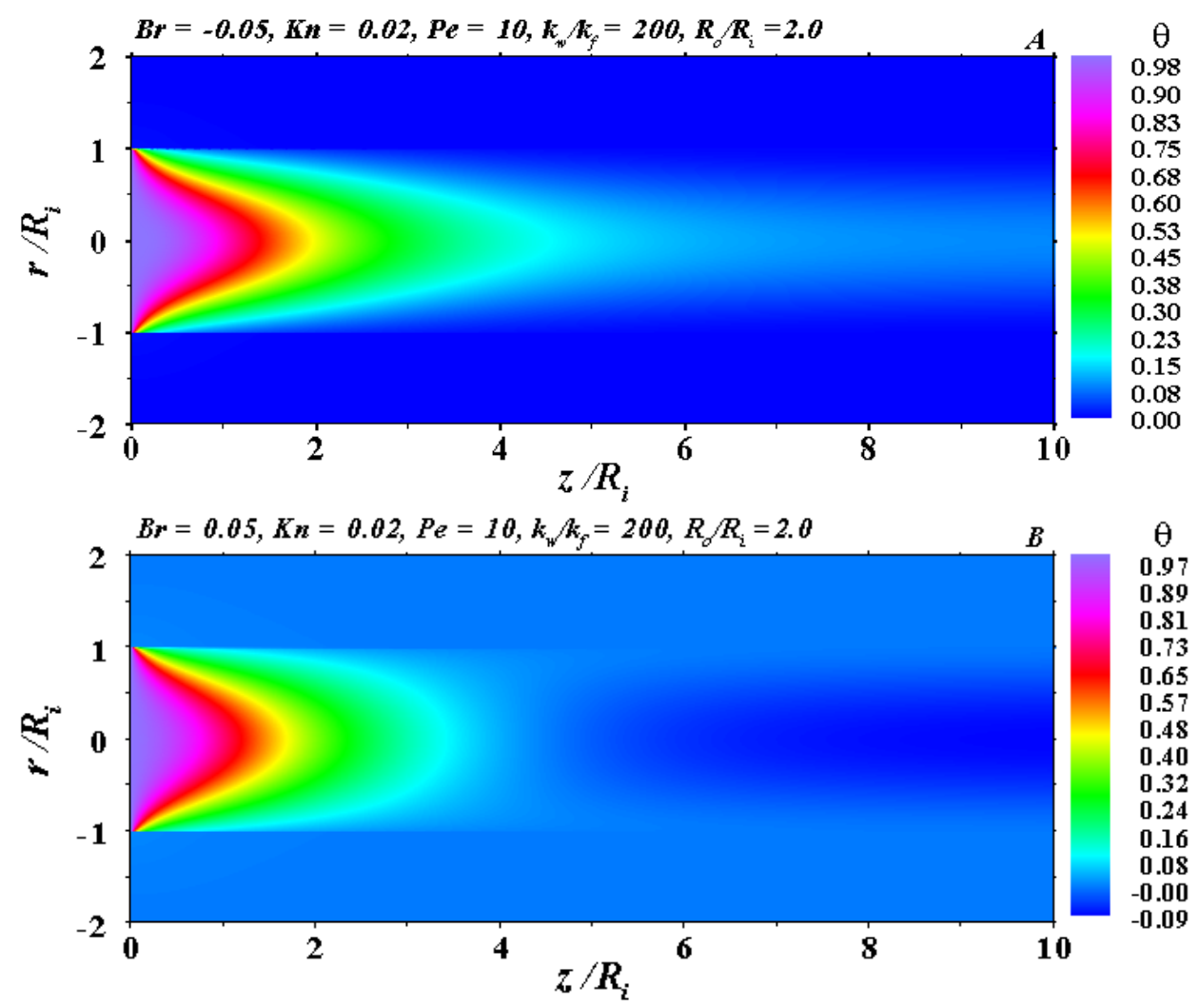

Fig. 11 Temperature contours in the whole domain for gas heating (A) and cooling (B).
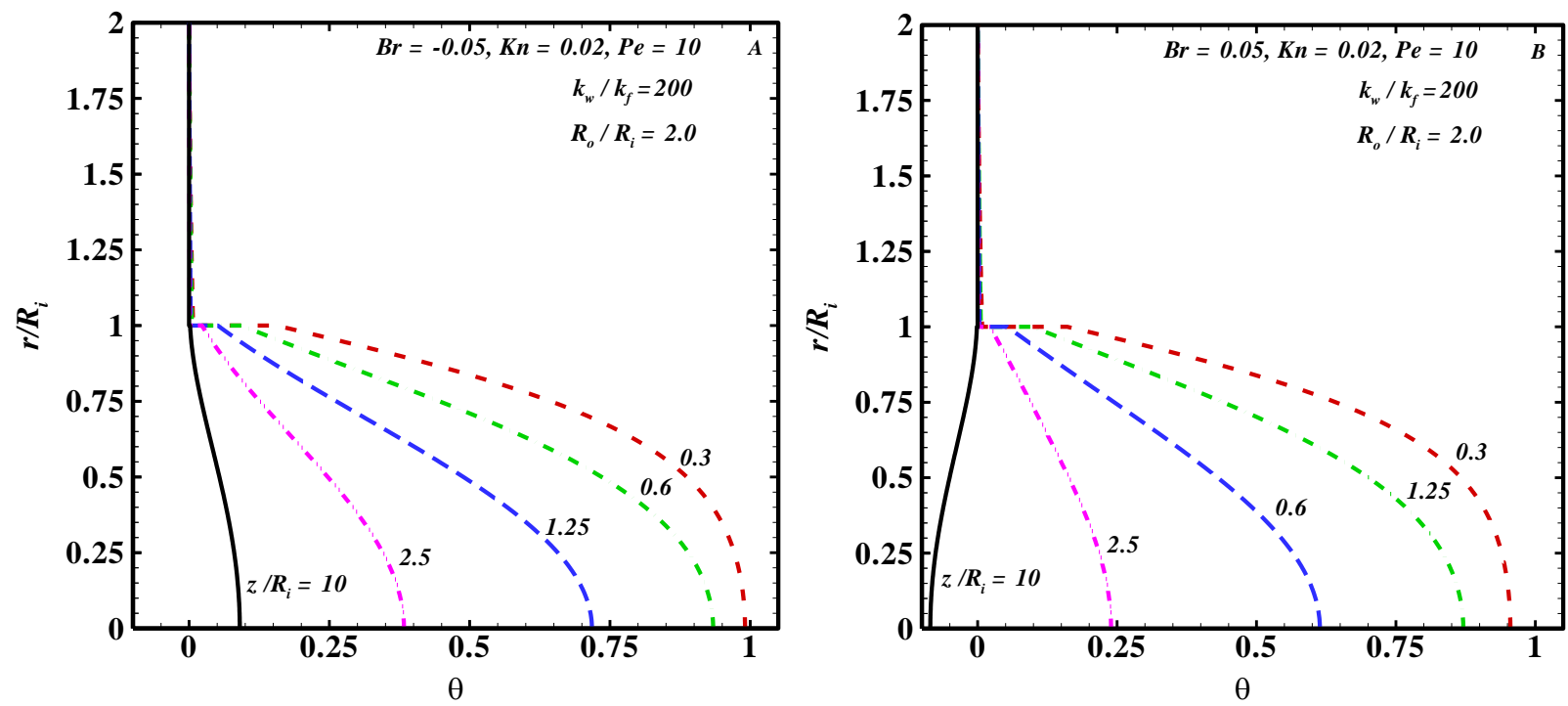

Fig. 12 Temperature profiles in the upper half domain at different axial locations for gas heating (A) and cooling (B).

\section{Conclusions}

Conjugate heat transfer in a microtube gas flow is studied numerically, considering gas rarefaction, axial and radial conduction in both the tube wall and the gas, as well as the shear work and the combined viscous dissipation and pressure work effects. The numerical results are verified against data available in the literature. Effects of different parameters on heat transfer are analyzed, particularly the tube wall thickness and the wall to gas thermal conductivity ratio, in addition to the effects of Peclet and Brinkman numbers in the presence of the wall. The results are compared with the zero wall thickness case wherever appropriate.

Heat transfer characteristics in microtube gas flow are shown to be dependent on rarefaction, wall conduction, 
axial conduction in the gas, shear work and the combined viscous dissipation and pressure work. The analysis shows that heat exchange between the tube wall and the gas takes place only in the thermally developing region. Therefore, for efficient heat exchange between the gas and the tube wall, the tube length may not need to exceed the thermal entrance length. A shorter tube length also implies reducing the frictional losses. The thermal entrance length and other heat transfer parameters are however dependent on the flow variables as well as on the tube wall heat conduction. Higher tube wall thickness and lower wall thermal conductivity reduces the heat transfer between the gas and the wall due to the higher thermal resistance. Higher rarefaction in the gas decreases the heat exchange between the tube wall and the gas. The higher the Peclet number (i.e., lower axial conduction in the fluid), the higher the Nusselt number and the inner wall heat flux. The effect of the tube wall thickness with insulated tube ends becomes vanishingly small when $k_{w} \gg k_{f}$, hence, the choice of the tube thickness becomes more relevant to rigidity and fabrication considerations, not to heat transfer.

\section{Nomenclature}

a A constant compression parameter

A Area $\left(m^{2}\right)$

$b \quad$ Tube wall thickness to its inner radius ratio

$\mathrm{Br} \quad$ Brinkman number $\left(=\mu u_{m}^{2} /\left[k_{f} /\left(T_{o}-T_{w o}\right)\right]\right)$

$c_{p} \quad$ Specific heat $(\mathrm{J} / \mathrm{kg} . \mathrm{K})$

$D_{i} \quad$ Tube inner diameter $(m)$

$k \quad$ Thermal conductivity $(W / m . K)$

$K n \quad$ Knudsen number $\left(=\lambda / D_{i}\right)$

$N u_{t h} \quad$ Nusselt number $\left(=q_{c} D_{i} /\left[k_{f} /\left(T_{w i}-T_{m}\right)\right]\right)$

$N u_{\text {tot }} \quad$ Nusselt number $\left(=q_{w i} D_{i} /\left[k_{f} /\left(T_{w i}-T_{m}\right)\right]\right)$

$P \quad$ Pressure $\left(\mathrm{N} / \mathrm{m}^{2}\right)$

$P e \quad$ Peclet number $\left(=2 u_{m} R_{i} / \alpha\right)$

$\operatorname{Pr} \quad$ Prandtl number $(=v / \alpha)$

$q_{c} \quad$ Wall heat flux due to conduction $\left(\mathrm{W} / \mathrm{m}^{2}\right)$

$Q_{c} \quad$ Dimensionless wall heat flux due to conduction

$q_{w i} \quad$ Wall heat flux due to both conduction and shear work $\left(\mathrm{W} / \mathrm{m}^{2}\right)$

$Q_{w i} \quad$ Dimensionless wall heat flux due to both conduction and shear work

$R_{i} \quad$ Microtube inner radius $(m)$

$R_{o} \quad$ Microtube outer radius $(m)$

$T \quad$ Temperature $(K)$

$T_{m} \quad$ Mean temperature $(K)$

$T_{o} \quad$ Inlet temperature $(K)$

$T_{w i} \quad$ Inner wall temperature $(K)$

$u \quad$ Velocity component in the $z$-direction $(\mathrm{m} / \mathrm{s})$

$z, r \quad$ Coordinates (m)

\section{Greek symbols}

$\alpha \quad$ Thermal diffusivity $\left(\mathrm{m}^{2} / \mathrm{s}\right)$

$\beta_{V} \quad$ A constant parameter, $\beta_{V}=\left(2-\sigma_{V}\right) / \sigma_{V}$

$\beta_{T} \quad$ A constant parameter, $\beta_{T}=\left(2-\sigma_{T}\right) / \sigma_{T}$

$\beta_{T}^{*} \quad$ A constant parameter

$\gamma \quad$ Specific heat ratio

$\xi \quad$ Dimensionless axial distance, $\xi=z / R_{i}$

$\eta \quad$ Dimensionless distance, $\eta=r / R_{i}$

$\theta \quad$ Dimensionless temperature, $\theta(\xi, \eta)=\left(T-T_{w o}\right) /\left(T_{o}-T_{w o}\right)$

$\lambda \quad$ Fluid mean free path $(m)$

$\rho \quad$ Density $\left(\mathrm{kg} / \mathrm{m}^{3}\right)$

$\sigma_{T} \quad$ Thermal accommodation coefficient

$\sigma_{V} \quad$ Momentum accommodation coefficient 
$\begin{array}{ll}v & \text { Kinematic viscosity }\left(\mathrm{m}^{2} / \mathrm{s}\right) \\ \mu & \text { Dynamic viscosity }\left(\mathrm{N} . \mathrm{s} / \mathrm{m}^{2}\right)\end{array}$

\section{Subscripts}

c Conduction

$f \quad$ Fluid

fd Fully developed

th Thermal

tot Total

$w \quad$ Wall

wi Inner wall side

wo Outer wall side

$m \quad$ Mean value

sw Shear work

\section{References}

Avci, M., Aydın, O. and Arici, M. E., Conjugate heat transfer with viscous dissipation in a microtube, International Journal of Heat and Mass Transfer, Vol. 55, No. 19-20 (2012), 5302-5308.

Aydın, O. and Avcı, M. (2006). Heat and fluid flow characteristics of gases in micropipes, International Journal of Heat and Mass Transfer, Vol. 49, No. 9-10 (2006), pp. 1723-1730.

Barışık, M., Yazıcıoğlu, A. G., Çetin, B. and Kakaç, S., Analytical solution of thermally developing microtube heat transfer including axial conduction, viscous dissipation, and rarefaction effects. International Communications in Heat and Mass Transfer, Vol. 67 (2015), pp. 81-88.

Cole, K. D., Cetin, B. and Brettmann, L., Microchannel Heat Transfer with Slip Flow and Wall Effects, Journal of Thermophysics and Heat Transfer, Vol. 28, No. 3 (2014), pp. 455-462.

Darici, S., Bilir, S. and Ates, A., Transient conjugated heat transfer for simultaneously developing laminar flow in thick walled pipes and minipipes, International Journal of Heat and Mass Transfer, Vol. 84 (2015), pp. 1040-1048.

Faghri, M. and Sparrow, E. M., Simultaneous Wall and Fluid Axial Conduction in Laminar Pipe - Flow Heat Transfer, ASME Journal of Heat Transfer, Vol. 102, No. 1 (1980), pp. 58-63.

Jeong, H.-E. and Jeong, J.-T., Extended Graetz Problem Including Axial Conduction and Viscous Dissipation in Microtube, Journal of Mechanical Science and Technology, Vol. 20, No. 1 (2006), pp. 158-188.

Kabar, Y., Bessaih, R. and Rebay, M., Conjugate heat transfer with rarefaction in parallel plates microchannel, Superlattices and Microstructures, Vol. 60 (2013), pp. 370-388.

Knupp, D. C., Cotta, R. M. and Naveira-Cotta, C. P., Theoretical analysis of conjugated heat transfer with a single domain formulation and integral transforms, International Communications in Heat and Mass Transfer, Vol. 39, No. 3 (2012), pp. 355-362.

Knupp, D. C., Cotta, R. M. and Naveira-Cotta, C. P., Conjugated Convection-Conduction Analysis in Microchannels With Axial Diffusion Effects and a Single Domain Formulation, ASME Journal of Heat Transfer, Vol. 135, No. 9 (2013), 091401.

Knupp, D. C., Cotta, R. M., Naveira-Cotta, C. P. and Kakac, S., Transient conjugated heat transfer in microchannels: Integral transforms with single domain formulation," International Journal of Thermal Sciences, Vol. 88 (2015), pp. 248-257.

Kuo, J. C. and Lin, T. F., Steady Conjugate Heat Transfer in Fully Developed Laminar Pipe Flows, Journal of Thermophysics and Heat Transfer, Vol. 2, No. 3 (1988), pp. 281-283.

Lelea, D., The conjugate heat transfer of the partially heated microchannels, Heat and Mass Transfer, Vol. 44, No. 33 (2007), pp. 33-41.

Nonino, C., Savino,S, Giudice, S. D. and Mansutti, L., Conjugate forced convection and heat conduction in circular microchannels, International Journal of Heat and Fluid Flow, Vol. 30, No. 5 (2009), pp. 823-830.

Rahimi, M. and Mehryar, R., Numerical study of axial heat conduction effects on the local Nusselt number at the entrance and ending regions of a circular microchannel, International Journal of Thermal Sciences, Vol. 59 (2012), pp. 87-94. 
Ramadan, K., Effects of pressure work, viscous dissipation, shear work and axial conduction on convective heat transfer in a microtube, Case Studies in Thermal Engineering, Vol. 10 (2017), pp. 370-381.

Ramadan, K., Numerical Analysis of Conjugated Convection-Conduction Heat Transfer in a Microtube Gas Flow, Journal of Thermal Science and Engineering Applications, Vol. 11, No. 1 (2019a), 011004, Paper No: TSEA-18-1159; doi: 10.1115/1.4040991

Ramadan, K. M., Kamil, M. and Bataineh, M. S., Conjugate heat transfer in a microchannel simultaneously developing gas flow: A vorticity stream function-based numerical analysis. Journal of Thermal Science and Engineering Applications, Vol. 11, No. 6 (2019), 061011.

Sen, S., and Darici, S., Transient Conjugate Heat Transfer in a Circular Microchannel Involving Rarefaction, Viscous Dissipation and Axial Conduction Effects, Applied Thermal Engineering, Vol. 111(2017), pp. 855-862.

Schmidt, F. W. and Zeldin, B., Laminar Heat Transfer in the Entrance Region of Ducts, Applied Scientific Research, Vol. 23, No. 1 (1970), pp. 73-94.

Shah, R.K. and London, A.L, Laminar flow forced convection in ducts, in: Advances in Heat Transfer (1978), Academic Press, New York.

Zhang, L.-Z., 2014, Conjugate Heat and Mass Transfer in Heat Mass Exchanger Ducts (2014), Academic Press.

Zhang, S-X., He, Y-L., Lauriat, G. and Tao, W.-Q., Numerical studies of simultaneously developing laminar flow and heat transfer in microtubes with thick wall and constant outside wall temperature, International Journal of Heat and Mass Transfer, Vol. 53, No. 19-20 (2010), pp. 3977-3989. 